\title{
Oscillations of Large-Scale Structures in turbulent Mixed Convection in a rectangular enclosure
}

\author{
A. Westhoff, D. Schmeling, J. Bosbach and C. Wagner
}

German Aerospace Center (DLR) Göttingen - Institute of Aerodynamics and Flow Technology - Bunsenstr. 10, D-37073 Göttingen, Germany

andreas. westhoff@dlr.de

\section{Introduction}

The term mixed convection (MC) is used to describe the process of heat transfer in fluids where forced convection (FC) and thermal convection (TC) coexist. Mixed convection is an often occurring flow condition e.g. in the oceans, atmosphere, indoor climatisation or industrial processes and applications [1]. In many flow situations convection is the prevalent transport mechanism of heat whereas the heat transfer strongly depends on the dynamics of the largescale structures. In this study we investigate the formation of large-scale circulation (LSC) in mixed convection and the influence of the dynamics of the LSC, also known as mean wind, on the heat transfer. Measurements of the heat transfer $\dot{Q}(t) \sim\left(T_{\text {out }}(t)-\bar{T}_{\text {in }}\right) / \Delta T\left(T_{i n}\right.$ : temperature of the flow at the inlet, $T_{\text {out }}$ : temperature of the flow at the outlet) in mixed convection revealed very low frequency oscillations for $\dot{Q}[5]$, which strongly depend on the magnitude of the Reynolds number $R e$ and Rayleigh number $R a$ as well as on the Archimdes number $A r$.

For the investigations presented here a simple rectangular container in which a fluid layer is confined between two isothermal horizonal plates heated from below, cooled from above and can be exposed to forced convection was chosen. The problem is defined by five dimensionless parameters, i.e. the Rayleigh number $R a \equiv \Delta T \beta g H^{3} / \kappa \nu$, the Reynolds number $R e \equiv U H / \nu$, the Prandtl number $\operatorname{Pr} \equiv \nu / \kappa$ and the aspect ratios of the rectangular container $\Gamma_{\mathrm{xz}} \equiv W / H$ and $\Gamma_{\mathrm{yz}} \equiv L / H$. Here $\beta$ denotes the isobaric thermal expansion coefficient, $g$ the acceleration due to gravity, $\Delta T$ the applied temperature difference, $\kappa$ the thermal diffusivity, $\nu$ the kinematic viscosity, $U$ the characteristic velocity, $W$ the width, $L$ the length and $H$ the height of the cell. An additional parameter used for mixed convection is the Archimedes number $A r=R a /\left(R e^{2} \times P r\right)=\Delta T \beta g H^{3} / U^{2}$ which is the ratio of buoyancy 
and inertia forces. For $A r \ll 1$ the flow is primarily driven by inertia forces, whereas for $A r \gg 1$ the flow is dominated by buoyancy forces.

\section{Experimental set up}

To cover a large parameter range of $600<R e<3 \times 10^{6}$ and $1 \times 10^{5}<$ $R a<1 \times 10^{11}$ two convection cells have been constructed using air as working fluid under different pressure conditions with an aspect ratio of $\Gamma_{\mathrm{xy}}=1$ and $\Gamma_{\mathrm{xz}}=5$. The cells are equipped with an air inlet at the top and an air outlet at the bottom. The in- and outlet channels are located at the same side of the cell, are rectangluar and span the whole length of the cell. The inlet channel has a height of $H_{\text {in }}=\frac{1}{20} \times H$ and a length of $L_{\text {in }}=30 \times H_{\text {in }}$ to assure a well defined fully developed channel flow at the cell inlet, while the outlet channel has a height of $H_{\text {out }}=\frac{3}{5} \times H_{\text {in }}$ and a length of $L_{\text {out }}=30 \times H_{\text {out }}$. All side walls are thermally insulated by a layer system with an insulating sheath of air between two transparent windows. Hence we nearly realise adiabatic boundary conditions while maintaining the optical accessibility of the cell. The bottom is equipped with a heating plate and the top with a heat exchanger with cooling fins. One of the cells with the dimensions $W \times H \times L=0.1 \mathrm{~m} \times 0.1 \mathrm{~m} \times 0.5 \mathrm{~m}$ was designed to be operated under high pressure conditions of up to 100 bar. The second convection cell has been designed to work under ambient pressure conditions with the same aspect ratio, but its dimensions are scaled by a factor of 5 .

In this article particle image velocimetry (PIV) results of the flow in the large cell under ambient pressure are presented, analysed and compared to results of temperature measurements conducted in the small cell at 10 bar. Two-dimensional two-component (2D2C) PIV has been carried out in different cross sections. Additionally two-dimensional three-component (2D3C) PIV has been performed in a longitudinal cross scection. The 2D2C measurement planes are located at $0.5 \times L, 0.375 \times L, 0.25 \times L$ and $0.1 \times L$ while the $2 \mathrm{D} 3 \mathrm{C}$ cross section is located at $0.5 \times W$. The instantaneous velocity fields have been acquired with a repetition rate of $2 / 3 \mathrm{~Hz}$. For the forced convection case 800 and for the mixed convection case 4800 instantaneous velocity fields have been measured. Addionally results of a Proper Orthogonal Decomposion (POD) of the instantaneous velocity fields are presented, which have been calculated using the snapshots method by Sirovich [4].

\section{Results}

In the cell forced convection is induced by a pressure gradient between the inand outlet. As a result a nearly two-dimensional role structure develops, with a core which is stationary (Fig. 1). The mean wind behaves in the core like a solid body rotation (Rankine vortex) and the angular frequency is found 
to be $\omega_{\mathrm{FC}}=0.32 \mathrm{~s}^{-1}$. The other mechanism that drives the heat transport in mixed convection is the buoyancy force. In the buoyancy driven case hot plumes are rising from the bottom thermal boundary layer and cold plumes are descending from the top. As a result of this plume-motion coherent flow structures develop and form a LSC [3]. In the here used enclosure the plumemotion induces four LSCs, which are arranged in longitudinal direction. One of these LSCs is presented in Fig. 1 in terms of the time averaged velocity fields. Additionally, the contours in Fig. 1 depict the out of plane velocity component $w$. At the top (coloured red) the air which enters the cell through the inlet and at the bottom (coloured blue) the flow in direction of the outlet dominate.
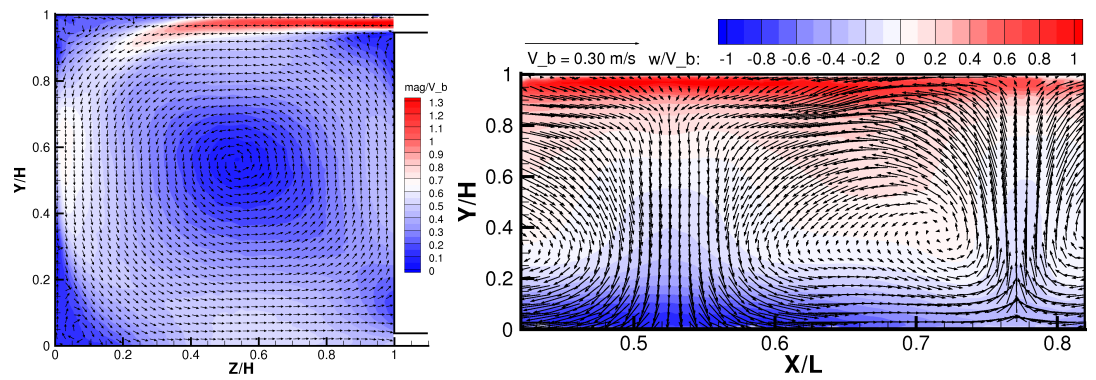

Fig. 1. Left: Time averaged velocity field of forced convection at $A r=0$ with $R e=1.0 \times 10^{4}$ at $0 . t 5 \times L$. Colour coded normalised velocity magnitude. Right: Time averaged velocity field of the 2D3C-PIV measurement for mixed convection at $A r=3.3$ with $R a=2.4 \times 10^{8}, R e=1.0 \times 10^{4}$ at $0.5 \times W$. All velocities are normalised with the buoyancy velocity $V_{b}=\sqrt{\beta H^{3} \Delta T g}$. Only every fifth velocity vector is plotted.

Analysing the measured temperature data spatial temperature variations over the length of the outlet depending on the orientation of the buoyancy induced LSCs have been found. In regions of rising plumes $T_{\text {out }}$ is elevated and in regions where plumes are falling a lower $T_{\text {out }}$ can be observed. For cases $A r>1$, the temperature signal locally fluctuates in time (subplot Fig. 2). The corresponding powerspectrum of the local temperature fluctuations at $0.5 \times L$ (Fig. 2) reveals two characteristic frequencies. One of these characteristic frequencies is $\omega=0.32 \mathrm{~s}^{-1}$, which equals the angular frequency $\omega_{\mathrm{FC}}=0.32 \mathrm{~s}^{-1}$ of the role structure found in the PIV results of forced convection in the large cell. Although the temperature measurements were performed at 10 bar (small cell experiment), the same frequency appears and we assume this frequency to be associated with the dynamics of the role structure induced by forced convection. The second characteristic frequency $\omega_{\text {low }}=0.008 \mathrm{~s}^{-1}$ (Fig. 2) is much lower then $\omega_{\mathrm{FC}}$ and the angular frequency associated with the thermal convection induced LSCs $\omega_{\mathrm{TC}} \approx 0.5 \mathrm{~s}^{-1}$. 

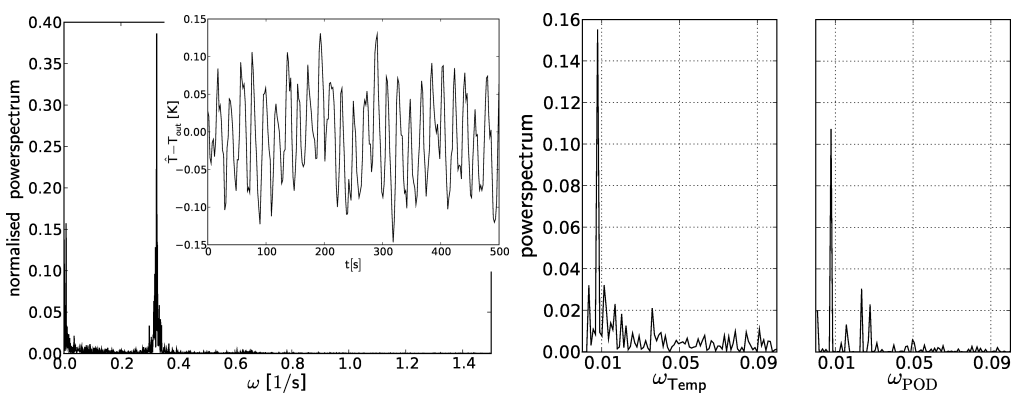

Fig. 2. Left: Powerspectrum of $T_{\text {out }}$ at $0.5 \times L$ for $A r=3.3, R a=2.4 \times 10^{8}$ and $R e=1 \times 10^{4}$ at 10 bar. The subplot shows the first 500 of $5000 \mathrm{~s}$ of the time series of the outlet temperature $(0.5 \times L)$. Right: Powerspectrum of the low frequency oscillations of $T_{\text {out }}$ and the powerspectrum of $\underline{\zeta}_{1}^{\mathrm{MC}}$.

To investigate if the above frequencies are related to the dynamics of any characteristic flow structures a POD analysis of the 2D3C PIV data obtained for forced and mixed convection has been performed. For both, forced and mixed convection, eigenfunctions with eigenvalues $\lambda_{1}^{\mathrm{MC}}=73 \%$ and $\lambda_{1}^{\mathrm{FC}}=90$ $\%$ of the total energy were obtained. Additionally the coefficient of the eigenfunction $\zeta$ which corresponds to the heighest eigenvalue has been analysed. The powerspectrum $\underline{\zeta}_{1}$ of the forced convection case reveals no characteristic frequency contrarily to the powerspectrum of the mixed convection case. The latter contains the same low characteristic frequency that has been found in the temperature measurements at 10 bar. Due to the concurrence of these frequencies it is concluded that this low frequency oscillation in the heat transfer is the result of the dynamics of the buoyancy induced LSCs. Even more, we assume that the oscillations are a result of torsional oscillations of the buoyancy induced LSCs in agreement with findings in Rayleigh-Bénard convection by Funfschilling et al. [2].

\section{References}

1. P. F. Linden, The fluid mechanics of natural ventilation, Annu. Rev. Fluid Mech., 31, 201, 1999.

2. D. Funfschilling, E. Brown and G. Ahlers, Torsional oscillations of the largescale circulation in turbulent Rayleigh-Bénard convection, J. Fluid Mech., 607, 119,2008

3. X.-L. Qui and P. Tong, Large-scale velocity structures in turbulent thermal convection, Phys. Rev. E, 64, 036304, 2001.

4. L. Sirovich. Turbulence and the dynamics of coherent structures part ii: Symmetries and transformation, Quarterly of Applied Mathematics, 45(3), 573, 1987.

5. A. Westhoff, J. Bosbach, and C. Wagner. Scaling of mixed convection in aircraft cabins. 26th International Congress of the Aeronautical Sciences, 2008. 